\title{
Diabetes mellitus accelerates the progression of osteoarthritis in streptozotocin-induced diabetic mice by deteriorating bone microarchitecture, bone mineral composition, and bone strength of subchondral bone
}

\author{
Hua-Jun Wang ${ }^{1}$, Hugo Giambini ${ }^{2}$, Ji-Wen Chen ${ }^{1}$, Qiu-Shi Wang ${ }^{1}$, Hui-Ge Hou ${ }^{1}$, Si-Min Luo ${ }^{1}$, \\ Jun-Yuan Chen ${ }^{1}$, Teng-Feng Zhuang ${ }^{1}$, Yuan-Feng Chen ${ }^{1}$, Ting-Ting $\mathrm{Wu}^{1}$, Zhen-Gang Zha ${ }^{1}$, \\ You-Jie Liu ${ }^{1}$, Xiao-Fei Zheng ${ }^{1}$
}

${ }^{1}$ The First Clinical College, Jinan University \& Department of Orthopedic Surgery and Sports Medicine Center, The First Affiliated Hospital, Jinan University, Guangzhou, China; ${ }^{2}$ Department of Biomedical Engineering, The University of Texas at San Antonio, San Antonio, TX, USA

Contributions: (I) Conception and design: HJ Wang, H Giambini, YJ Liu, XF Zheng; (II) Administrative support: HJ Wang, H Giambini, YJ Liu, XF Zheng; (III) Provision of study materials or patients: HJ Wang, H Giambini, YJ Liu, XF Zheng; (IV) Collection and assembly of data: HJ Wang, JW Chen, QS Wang, HG Hou, SM Luo, JY Chen, TF Zhuang; (V) Data analysis and interpretation: HJ Wang, YF Chen, TT Wu, ZG Zha; (VI) Manuscript writing: All authors; (VII) Final approval of manuscript: All authors.

Correspondence to: Xiao-Fei Zheng; You-Jie Liu. The First Clinical College, Jinan University \& Department of Orthopedic Surgery and Sports Medicine Center, The First Affiliated Hospital, Jinan University, Guangzhou 510632, China. Email: zhengxiaofei12@163.com; lyjlovesjy0204@gmail.com.

Background: The purpose of this study was to develop an optimal diabetes-osteoarthritis (DM-OA) mouse model to validate that diabetes aggravates osteoarthritis $(\mathrm{OA})$ and to evaluate the microarchitecture, chemical composition, and biomechanical properties of subchondral bone (SB) as a consequence of the DM-OAinduced damage induced.

Methods: Mice were randomly divided into three groups: DM-OA group, OA group, and sham group. Blood glucose levels, body weight, and food intake of all animals were recorded. Serum calcium (Ca) and osteocalcin (OCN) levels were compared in the three groups. The messenger ribonucleic acid (mRNA) and protein expression of key regulators for bone metabolism were detected. A semi-quantitative grading system [Osteoarthritis Research Society International (OARSI)] was used to evaluate cartilage and SB degeneration. Microspectroscopy, microindentations, micro-computed tomography (CT) imaging, and fracture load of compression testing were also used to evaluate trabecular SB properties.

Results: Glycemic monitoring and pancreas pathological results indicated stable high blood glucose and massive destruction of pancreas and islet cells in the DM-OA group. Serum levels of bone specific alkaline phosphatase (ALP-B) and tartrate-resistant acid phosphatase 5b (TRACP-5b) in the DM-group were higher than those of the other two groups while levels of serum $\mathrm{Ca}$ and OCN were lower. Meanwhile, the protein and mRNA expression of osteoblast-specific biomarkers [osteoprotegerin/receptor activator of nuclear factor kappa-B ligand (OPG/RANKL) ratio, collagen type I (COL-I), Runt-related transcription factor 2 (RUNX-2), OCN] were suppressed, and osteoclast-specific biomarkers [sclerostin (SOST)] was elevated in the DM-OA group. The mineral-to-collagen ratio, microindentation elastic modulus, hardness, micro-architectural parameters, bone mineral density, and fracture load of SB trabecular bone of the DM-OA group joint were lower than those of the other two groups. On the other hand, The OARSI score, trabecular spacing, and structural model index of the DM-OA group joint were higher than those of the other two groups.

Conclusions: The glycemic and pancreatic pathological results indicated that the DM-OA model was a simple and reliable model induced by streptozotocin (STZ) and surgery. The results revealed the mechanisms through which diabetes accelerates $\mathrm{OA}$; that is, by damaging and deteriorating the functions of SB, including its microarchitecture, chemical composition, and biomechanical properties. 
Keywords: Diabetes mellitus (DM); osteoarthritis (OA); subchondral bone (SB); bone strength

Submitted Oct 10, 2020. Accepted for publication Feb 21, 2021.

doi: 10.21037/atm-20-6797

View this article at: http://dx.doi.org/10.21037/atm-20-6797

\section{Introduction}

Osteoarthritis (OA) is the most common form of degenerative joint disorder. It is forecasted that $25 \%$ of the adult population ( $80 \%$ of the geriatric population $>60$ years), or more than 50 million people in the US, will be affected by this disease by $2020(1,2)$. OA, a multifactorial disease, is characterized by pain, stiffness, and functional impairment, ultimately resulting in disability and a poor quality of life. Although the etiology is unclear, joint components are presently considered to be involved in OA progression. The accumulative evidence suggests that subchondral bone $(\mathrm{SB})$ plays a crucial role in the initiation and progression of OA (3), which manifests as dynamic remodeling in parallel with (or even preceding) cartilage degradation (4-6). The microarchitectural and histopathological changes deteriorate physiological function, SB integrity, and the interaction (crosstalk) between cartilage and SB, which accelerates OA progression. Heterogeneities in the architecture, density, and rigidity of SB in OA and reduced elasticity result in abnormal tensile stress and shear stress towards the articular cartilage, thus facilitating secondary cartilage damage degeneration (7). Recent animal and clinical trials have demonstrated that targeting SB via bone protective agents can break this vicious circle, relieve joint pain, and slow articular cartilage degeneration (5). Therefore, SB is currently recognized as a key to developing targeted and effective therapeutic strategies to treat OA.

Similarly, diabetes mellitus (DM) is also a common chronic disease with high morbidity and disability threatening global public health. Interestingly, DM and OA share common risk factors, including age, diet, obesity, lifestyle, and multifactorial etiology, which are characterized by a combination of genetic and environmental factors. Accumulating epidemiological and experimental findings have shown that DM is an independent risk factor and prognostic predictor for OA (8-12). A cross-sectional study of the US Third National Health and Nutrition Examination Survey (NHANES III) has demonstrated that $11 \%$ of the patients with OA suffered DM, which was a significantly higher prevalence than that of the general population (10). Furthermore, a 20-year longitudinal cohort study of 927 individuals revealed that DM has an independent role in pathogenesis and OA advancement (9). Recently, the epidemiological association between OA and $\mathrm{DM}$ has been further confirmed in two independent meta-analyses. These studies showed that more substantial evidence is accumulating in favor of an independent contribution of DM to OA development and progression (13-15). However, the underlying pathogenesis and connection between both diseases remain unclear. There is also an unfortunate paucity of quality research studies in animal models of DM relevant to OA (16). Recent statistics show that DM affects 382 million adults globally. This number is estimated to rise to 592 million by 2035 , which means the prevalence of DM-OA will be widely and abruptly growing worldwide (17-19). A better understanding of the mechanisms of diabetic OA is essential to provide opportunities to identify new therapeutic targets that might prevent and predict this disease.

It has been well established that DM extremely and negatively affects bone condition. It is advocated that DM be incorporated as a risk factor for fractures at all ages in male and female individuals, increasing further above that of the general population with aging (20). Specifically, in subjects with type $1 \mathrm{DM}$ (T1DM), there is approximately a 6-time increase in hip fracture risk and a two-fold increase in vertebral fracture risk compared to individuals without DM (21). Type 2 DM (T2DM) is also associated with an about $2-/ 3$-fold increase in hip fracture risk and a $50-80 \%$ increased extremity fracture risk compared with nondiabetic subjects $(22,23)$. DM could impact bone quality and density by unbalancing bone metabolism processes and systems (24). Consequently, we hypothesized the DM could also interfere with SB remodeling and aggravate the severity of OA. Unfortunately, there is a lack of quality research on DM's effect on SB and its role in DM-OA progression both in clinical and animal studies.

Therefore, we performed this comprehensive study to assess the changes in physiological properties and functions of SB and cartilage during the progression of DM-OA. This study had three objectives: (I) to develop a DM-OA mouse 


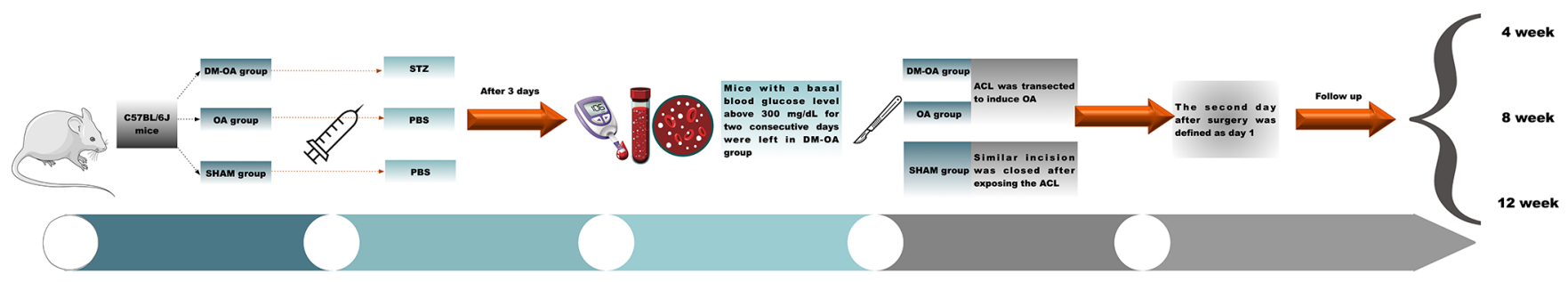

Figure 1 Timeline of the animal model and treatment before follow-up. The mice were divided randomly into three groups: diabetesosteoarthritis (DM-OA) group, osteoarthritis (OA) group, and sham group. After 1 week of acclimatization, the DM-OA group was injected intraperitoneally (IP) with $150 \mathrm{mg} / \mathrm{kg}$ streptozotocin (STZ) in a single injection to induce diabetes, while the OA and sham groups were given a PBS vehicle at the same time. On the third day after the STZ injection, blood glucose levels were measured. The anterior cruciate ligament (ACL) was transected to induce OA. Follow-up was conducted at 4, 8, and 12 weeks.

model that would allow for clinical and biological research; (II) to validate that DM aggravates $\mathrm{OA}$ pathogenesis in vivo; and (III) to evaluate the microarchitecture, chemical composition, and biomechanical properties of $\mathrm{SB}$ as a consequence of the damage induced by DM-OA. We present the following article following the ARRIVE reporting checklist (available at http://dx.doi.org/10.21037/ atm-20-6797).

\section{Methods}

\section{Animal model and treatment}

Eight-week-old male C57BL/6J mice from Guangdong Medical Lab Animal Center (Guangzhou, China) were used in the present study. The mice were randomly divided into three groups: DM-OA group, OA group, and sham group. After 1 week of acclimatization, the DM-OA group was injected intraperitoneally (IP) with $150 \mathrm{mg} / \mathrm{kg}$ streptozotocin (STZ, Sigma, St. Louis, MO, USA) in a single injection to induce DM, while the $\mathrm{OA}$ and sham groups were given a phosphate-buffered saline (PBS) vehicle at the same time. On the third day after STZ injection, blood glucose was measured in whole blood collected from the tail vein by a portable glucometer (Accu-Check Active, Roche Diagnostics Limited, Germany). Only mice with basal glucose above $300 \mathrm{mg} / \mathrm{dL}$ for two consecutive days were considered to be diabetic and were included in the present study; the remaining mice in the DM-OA group were excluded.

After quarantine and conventional health measures, OA was surgically induced in the DM-OA and OA groups by transection of the anterior cruciate ligament (ACLT) of the right knee, as previously described (25). Briefly, the ACL was transected to induce abnormal mechanical loading- associated $\mathrm{OA}$ and to ensure that no other components were damaged. For the sham group, a similar incision was closed after exposing the ACL, which was used as the control. Mice were observed daily to verify healing and to ensure that they were using their right limb.

The second postoperative day was defined as day 1 . The weekly blood glucose, body weight, and food intake of all animals were recorded during the entire experimental period. The animals were group-housed in temperaturecontrolled quarters $\left(20-22^{\circ} \mathrm{C}\right)$ according to a 12-hour light/ dark cycle and had ad libitum access to water and standard chow. All cages contained wood shavings, bedding, and a cardboard tube for environmental enrichment. Experiments were performed under a project license (No. 20160223001) granted by the Animal Care and Experiment Committee of Jinan University School of Medicine. All animals were kept in strict accordance with the National Laboratory Animal Management Regulations and guidelines of the Animal Feeding and Ethics Committees of the Experimental Animal Center of Jinan University (Figure 1, timeline of the study).

\section{Follow-up}

Animals were sacrificed at 4,8 , and 12 weeks postoperatively. After overnight fasting and the mild ether anesthesia, the blood and pancreas were sampled. Blood samples were withdrawn by venipuncture from the retrobulbar veins into plastic tubes with appropriate anticoagulant [ethylenediaminetetraacetic acid (EDTA)] to measure glycated hemoglobin (GHb, HbA1c), and serum samples without anticoagulant to measure glucose using an automatic analyzer (Roche Cobas 6000 c501, Temecula, CA, USA). The pancreas was placed in $4 \%$ 
Table 1 Primers sequence used for RT-PCR analysis

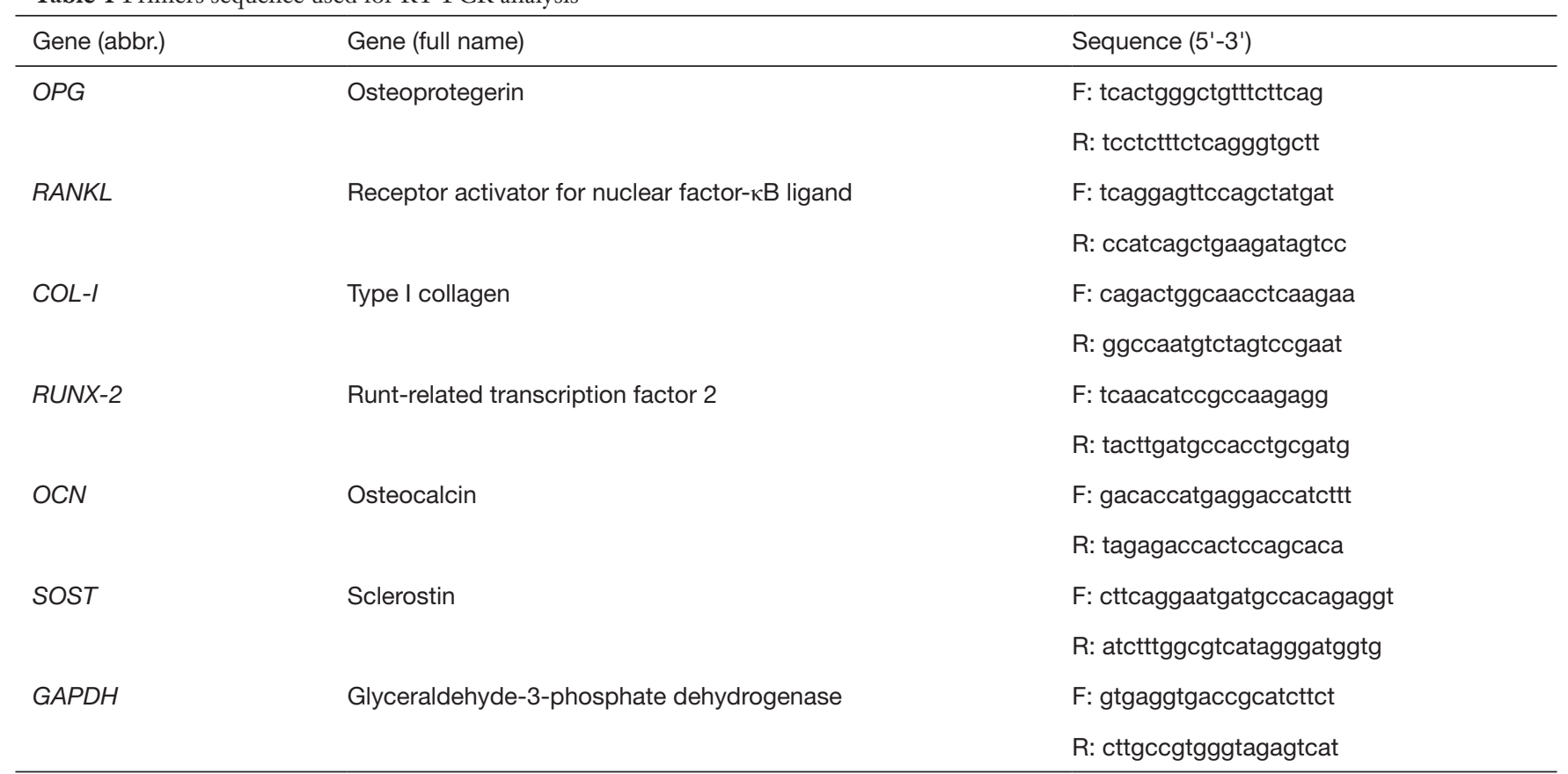

OPG, osteoprotegerin; RANKL, receptor activator of nuclear factor kappa-B ligand; COL-I, collagen type I; RUNX-2, Runt-related transcription factor 2; OCN, osteocalcin; SOST, sclerostin; GAPDH, glyceraldehyde-3-phosphate dehydrogenase.

paraformaldehyde and stained with hematoxylin-eosin (HE) for histopathological study.

The entire right knee joints from each group at every time point were dissected and fixed in $4 \%$ paraformaldehyde for $48 \mathrm{~h}$. The joints were then decalcified in $10 \%$ ethylenediaminetetraacetic acid for 3 weeks and embedded in the same paraffin block. Samples were cut into 5 - $\mu \mathrm{m}$ sections and prepared for histological and immunohistochemistry analysis to evaluate cartilage and SB degeneration. The other undecalcified joints were used to evaluate SB properties, including microcomputed tomography (CT) imaging, confocal Raman microspectroscopy, and microindentation.

\section{Intraperitoneal glucose tolerance test (IPGTT)}

Approximately 1 week before the termination, IPGTT was conducted to evaluate the glycogenic response to exogenously-administered glucose in unanaesthetized animals. After an overnight fast (12-16 hours), the mice were injected IP with $40 \%$ glucose $(2 \mathrm{~g} / \mathrm{kg}$ body weight). Tail blood samples were collected at $0,30,60$, and $120 \mathrm{~min}$ after glucose administration to measure glucose levels.

\section{Real-time polymerase chain reaction (RT-PCR)}

The tibias of all mice were crushed under liquid nitrogen conditions, and RNA extraction was performed following the TRIzol manufacturer's protocol (Invitrogen, Carlsbad, CA, USA). RNA integrity was verified by formaldehyde-agarose gel electrophoresis. $3 \mu \mathrm{g}$ of total RNA, Moloney murine leukemia virus reverse transcriptase (Invitrogen, Carlsbad, CA, USA), and oligo deoxythymidine (dT) (15) primers (Fermentas) were used to synthesize cDNAs (complementary deoxyribonucleic acid) by reverse transcription reactions. Regular PCR was then performed with the first strand of cDNA using a DNA Engine (ABI 7300). We used glyceraldehyde-3-phosphate dehydrogenase (GAPDH) to normalize the data. After denaturing cDNA at $95^{\circ} \mathrm{C}$ for $3 \mathrm{~min}$, the cycling conditions were set according to the kit instructions. The PCR primers used in this study are described in Table 1 .

\section{Western blotting}

The tibias were crushed under liquid nitrogen conditions, 
and the protein was extracted using the bone tissue protein extraction kit (Beijing Applygen Science Biotechnology; Beijing, China). Samples containing $30 \mu \mathrm{g}$ of protein were separated on $10 \%$ SDS-PAGE gel, transferred onto nitrocellulose membranes (Bio-Rad Laboratories, Hercules, CA, USA), and incubated in a blocking buffer ( $5 \%$ dried milk powder in TBS and $0.1 \%$ Tween 20). The membranes were incubated overnight at $4{ }^{\circ} \mathrm{C}$ using primary antibodies against osteoprotegerin (OPG) (ab203061) and receptor activator for nuclear factor $-\kappa$ B ligand (RANKL) (ab45039) from Abcam (Cambridge, UK), and Runtrelated transcription factor 2 (RUNX-2) (ab92336), COL1 (ab88147), sclerostin (SOST) (ab264040), and osteocalcin (OCN) (sc-365797) from Sant Cruz (Dallas, USA).

After three washes with TBST (Tris-buffered saline Tween-20), membranes were incubated with secondary antibody (donkey anti-mouse IgG, 1:10,000; goat anti-rabbit IgG, 1:4,000). The signal was visualized by the Odyssey Infrared Imaging System (LI-COR Biotechnology), and signals were quantified using the Odyssey Application 3.0 software and normalized with the corresponding GAPDH $(1: 5,000)$ as the internal control.

\section{Serum bone chemistries}

At 4, 8, and 12 weeks postoperatively, blood was collected and placed in EDTA tubes to prevent clotting. The serum was removed and stored at $-80{ }^{\circ} \mathrm{C}$. Calcium (Ca) concentrations were measured using commercial kits (Stanbio Laboratory, Boerne, USA). Serum tartrateresistant acid phosphatase 5b (TRACP-5b), OCN, and bone specific alkaline phosphatase levels (ALP-B) were measured using enzyme linked immunosorbent assay (ELISA) kits (Immutopics, Inc., San Clemente, CA, USA) according to the manufacturer's instructions.

\section{Histological analysis and assessment}

Knees were decalcified for 4 weeks in $4 \%$ paraformaldehyde and processed for standard paraffin embedding. Based on the Osteoarthritis Research Society International (OARSI) recommendations for the histological assessment of OA in the mice (24), three sections from each knee with 100 $\mu \mathrm{m}$ intervals were stained with safranin $\mathrm{O}$ and fast green. Cartilage damage was scored using the semi-quantitative grading and staging system [Pritzker et al. 2006 (26)]. Briefly, grade 0 corresponded to normal articular cartilage; grade 0.5 : loss of safranin-O and/or cellular changes without structural changes to the articular surface; grade 1 : small fibrillations without loss of cartilage; grade 2: vertical clefts down to the layer immediately below the superficial layer and some loss of surface lamina; grade 3: vertical clefts/erosion to the calcified cartilage extending to $<25 \%$ of the articular surface; grade 4: vertical clefts/erosion to the calcified cartilage extending to $25-50 \%$ of the articular surface; grade 5: vertical clefts/erosion to the calcified cartilage extending to $50-75 \%$ of the articular surface; grade 6: vertical clefts/erosion to the calcified cartilage extending $>75 \%$ of the articular surface. Grades were assigned to the medial and lateral tibial plateau and medial and lateral femoral condyles. Slides were blinded, and grades for all slides from each location of each sample were averaged. Grades from the three readers were averaged for each location.

\section{Micro-CT imaging and analysis}

To monitor the structural alterations and the SB tissue mineral content, we scanned the knee joints using microCT (Skysan, Belgium) with an isotropic voxel size of $9 \mu \mathrm{m}$ described previously. Regions of interest (ROI) within the trabecular bone restricted to the distal femoral epiphysis and proximal tibial epiphysis were created, and trabecular bone volume fraction (BV/TV), trabecular number ( $\mathrm{Tb}$. $\mathrm{N})$, trabecular separation (Tb.Sp), trabecular thickness (Tb.Th), trabecular mineralization density [bone mineral density (BMD)], and other trabecular bone parameters were determined using SkyScan software (Bruker, Massachusetts, USA). All measured parameters were represented as the mean \pm standard deviation (SD). Mimics software (Materialise, Plymouth, MI, USA) was used to create three-dimensional reconstructions for qualitative analysis.

\section{Confocal Raman microspectroscopy}

The chemical composition of the SB trabecular [mineralto-collagen, inorganic (mineral) to organic (matrix) ratio, $\mathrm{PO}_{4}^{3-} v_{1}$ /proline] was characterized by confocal Raman microspectroscopy (Jobin Yvon, Horiba, France) as described previously $(8,27)$. Joint samples were dehydrated in a graded alcohol series and embedded in polymethyl methacrylate (PMMA). The SB trabecular surface was exposed by slicing in the frontal plane using a hard tissue cutter (Exakt 300CP, Exakt Technologies, Germany). The section surface was ground on successive grits of 
silicon carbide paper and polished with 1-mm alumina slurry. The $\times 50$ objective focused the laser $(785-\mathrm{nm}$ laser diode source) on a $3-\mu \mathrm{m}$ region within the trabecular, and inelastic light was acquired by a spectrograph with $0.5 \mathrm{~cm}^{-1}$ spectral resolution. The measured spectra consisted of three accumulations with an integration time of $10 \mathrm{~s}$ each. Using vendor-supplied software (Jobin Yvon), background fluorescence in the spectra was subtracted by a modified polynomial fitting algorithm. Spectra were collected from 10 trabecular locations within the distal femoral epiphysis and proximal tibial epiphysis, respectively. The mineralto-collagen ratio was calculated as the $v_{1}$ phosphate peak intensity $\left(962 \mathrm{~cm}^{-1}\right)$ per proline peak intensity $\left(856 \mathrm{~cm}^{-1}\right)$ and averaged per bone.

\section{Microindentation testing}

SB trabecular material properties were analyzed with microindentation testing using the micro-indentation testing system (Microhardness testing machine, FUTURETECH CORP, Kanagawa, Japan) equipped with a Berkovich diamond tip indenter as previously described $(23,24)$. Briefly, samples were mounted and set in lowtemperature curing epoxy resin (EpoFix cold setting resin, Struers) and then ground through a silicon carbide series grinder followed by diamond polishing. Specimens were examined visually under an optical microscope, and at least 10 indentations were made on each sample. A typical indentation experiment resulted in a loading and unloading force-displacement curve. The Vickers diamond pyramidal indenter compressed at $25 \mathrm{~g}$ compression load onto the trabecular for a dwell time of $10 \mathrm{~s}$. An indentation mark was formed on the trabecular after loading. The indentation mark's diagonal length was measured immediately (within $1 \mathrm{~min}$ ) after the indentation to avoid the fast recovery of the indentation mark on the tissue matrix. The elastic properties were calculated between $50 \%$ and $95 \%$ of the initial unloading curve, and the Oliver-Pharr method was used to calculate elastic modulus and hardness.

\section{Mechanical compression of the $S B$}

The tibial epiphysis proximal portion was ground and mounted perpendicular to the tibial shaft for a punch-type compression test to measure the SB compression strength. The tibia was kept moist by wrapping in saline-soaked gauze from the time of collection until testing. The tibia was then positioned and centered under a $0.8-\mathrm{mm}$ diameter flat-tipped cylindrical punch fixed to the materials testing machine's actuator (Enduratec ELF 3200, Bose Corp., Eden Prairie, MN, USA). The axial compression was produced via the flat cylindrical punch at a $1 \mathrm{~mm} / \mathrm{min}$ displacement rate. During each test, the punch was centered on the trabecular bone while avoiding contact with the cortical bone. The displacement and compressive force were recorded until they peaked on the load-displacement diagram.

\section{Statistical analysis}

All data were presented as mean and SD and were analyzed using the Statistical Package for Social Sciences (SPSS, Chicago, IL, USA; version 22.0). Descriptive statistics were used to describe the demographic and measurement variables of all the scapulae. Categorical variables were expressed as frequencies and percentages for each variable. Continuous variables were presented as mean values $\pm \mathrm{SD}$. The factorial design analysis of variance (ANOVA) and Student-Newman-Keuls were applied to compare types. A $\mathrm{P}$ value $<0.05$ was considered statistically significant.

\section{Results}

\section{Induction of DM and physiological parameters in experimental animals}

Induction of DM with STZ was both effective and sustained for the DM-OA group. Glycemic monitoring showed that blood glucose markedly increased in the DM-OA group at all time points from 3 days after STZ administration (Figure 2A). Consistent with the elevation in blood glucose levels, the HbA1c level was also significantly higher in the DMOA group than the OA and sham groups $(8.24 \% \pm 0.46 \%$, $3.91 \% \pm 0.41 \%$, and $4.18 \% \pm 0.35 \%$, respectively) (Figure $2 B$ ). In contrast, the area under the receiver operating characteristic curve (AUC) analysis demonstrated an impaired glucose control for the DM-OA group by using an IPGT test. The mean AUC of the DM-OA group was significantly greater than that of the $\mathrm{OA}$ and sham groups $(\mathrm{P}<0.05)$ (Figure 2C). Pathological and histological results (Figure 2D) from the DMOA group indicated massive destruction of the pancreas, which exhibited decreased islets, damaged islet cytoarchitecture, and weak insulin staining of granulated $\beta$ cells compared with the OA and sham groups. No significant differences in the physiological parameters were observed between the OA group and the sham group. 
A
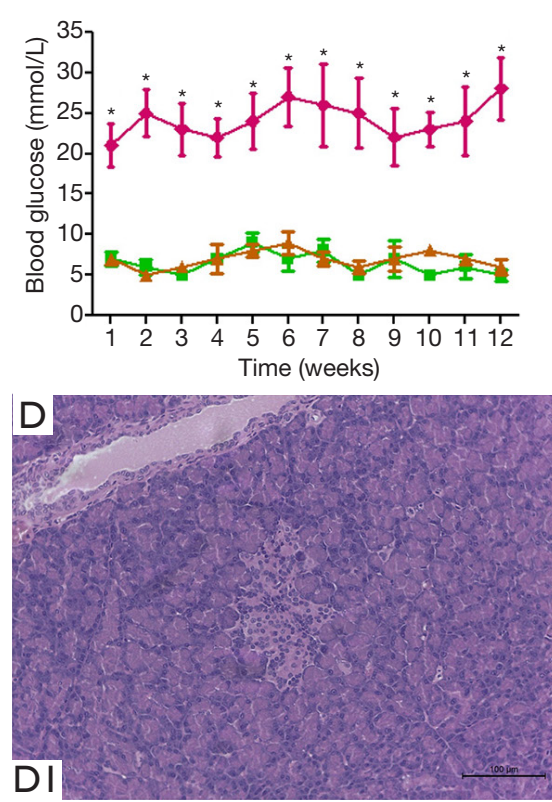

B

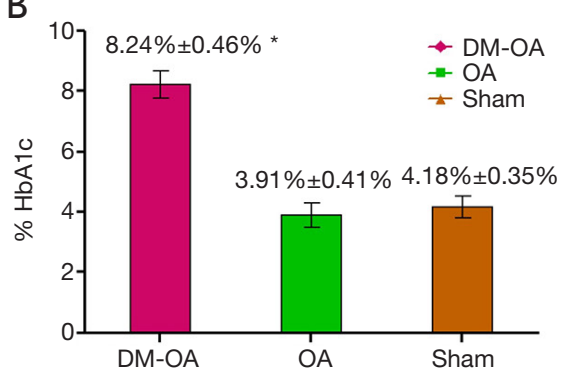

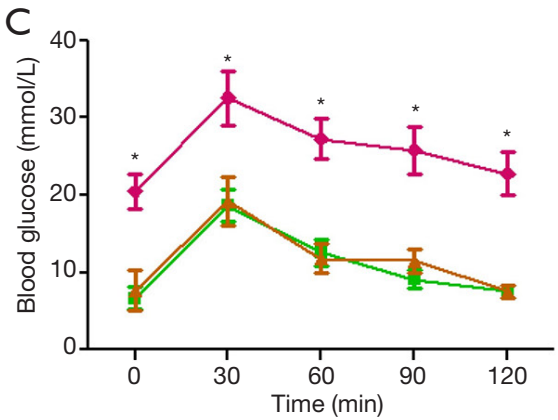
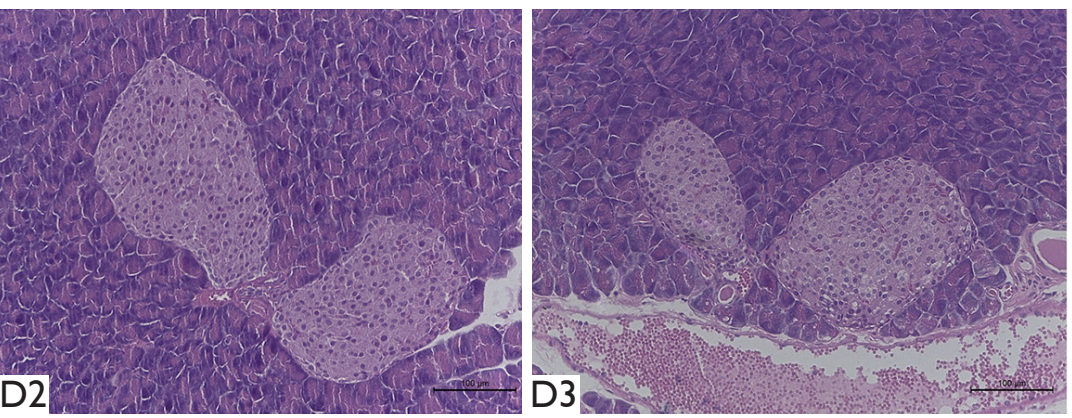

Figure 2 Induction of diabetes and physiological parameters in experimental animals. (A) After the injection of streptozotocin (STZ), the mean level of blood glucose was greater in the diabetes-osteoarthritis (DM-OA) group compared to the osteoarthritis (OA) and sham groups. *, $\mathrm{P}<0.05$. (B) HbA1c level was also significantly greater in the DM-OA group than OA and sham groups. *, $\mathrm{P}<0.05$. (C) The intraperitoneal glucose tolerance tests (IPGTTs) revealed a significant reduction in glycemic control in the DM-OA group. The area under the curve (AUC) was significantly greater in the DM-OA group. *, $\mathrm{P}<0.05$. (D) Histological study of the pancreas of the DM-OA group indicated a dramatic reduction in islets' size and cellularity. HE stained sections. (D1: DM-OA group; D2: OA group; D3 sham group, scale bar: 100 um). The data are expressed as the mean \pm SD and were compared using ANOVA and the Student-Newman-Keuls post-hoc test between the groups. *, $\mathrm{P}<0.05$ was considered significant.

\section{Serum bone chemistries}

Serum $\mathrm{Ca}$, and OCN levels were comparable in the three groups (Figure 3). At the 4-week stage of the study, the $\mathrm{Ca}$ and OCN levels were significantly different among the three groups. Compared with OA and sham groups, the $\mathrm{Ca}$ and OCN levels in DM-OA were significantly reduced at 8 and 12 weeks postoperatively (Figure 3B,D). Additionally, serum concentrations of TRACP-5b and ALP-B were determined. The serum levels of ALP-B and TRACP-5b in the DMOA group were significantly higher $(\mathrm{P}<0.05)$ than those of the other two groups from 8 to 12 weeks (Figure 3A,C). There was no significant difference between the OA group and the sham group in the serum chemical test.

\section{$m R N A$ and protein expression of regulators for bone metabolism}

To detect the change of osteoclast and osteogenic genes in
DM-OA, we measured the protein and mRNA expression, including OPG, RANKL, COL-I, RUNX-2, OCN, and SOST. The results of OPG and RANKL are expressed by the ratio (OPG/RANKL). The quantitative result showed that, in the DM-OA group, the ratio of OPG/RANKL and the expression of COL-I, RUNX-2, and OCN were significantly lower than that of the other two groups $(\mathrm{P}<0.05)$. In contrast, the expression of SOST was up-regulated in the DM-OA group $(\mathrm{P}<0.05)$ (Figure 4). Protein expression showed the same tendency as mRNA expression (Figure 5).

\section{Histological analysis and assessment}

OA was significantly accelerated in the DM-OA group assessed by histological analysis

The cartilage integrity in the tibial plateau and femoral condyle at 4,8 , and 12 weeks postoperatively was assessed histologically and scored according to the OARSI scoring system. As indicated by safranin-O/fast green staining 
A

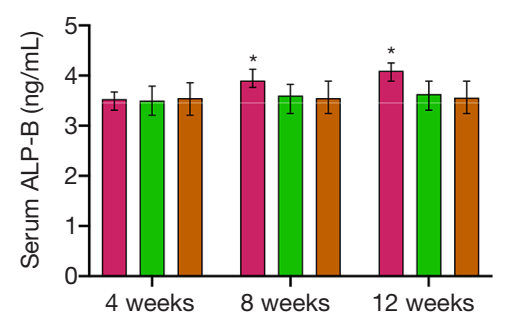

C

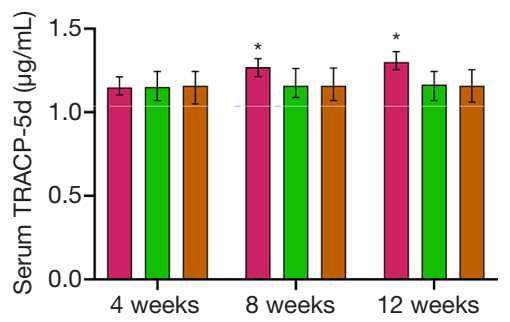

B

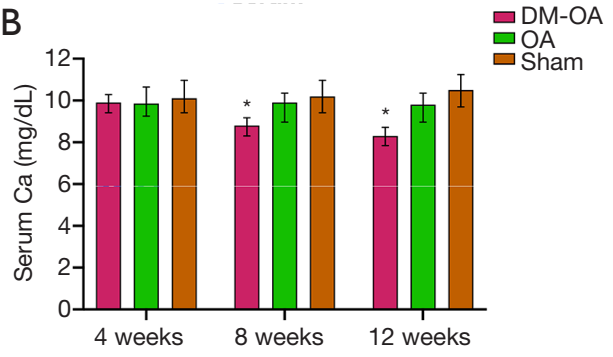

D

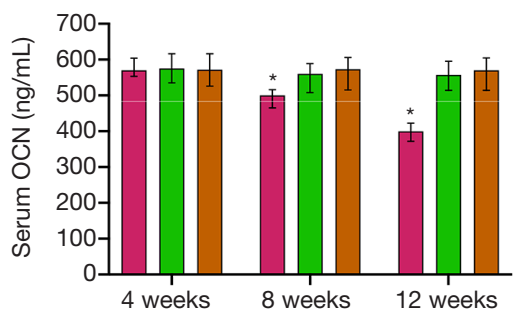

Figure 3 Serum biochemical parameter analysis. (A) Serum bone specific alkaline phosphatase (ALP-B); (B) serum calcium (Ca); (C) serum tartrate-resistant acid phosphatase 5b (TRACP-5b); (D) serum osteocalcin (OCN). The data are expressed as the mean \pm SD and were compared using ANOVA and the Student-Newman-Keuls post-hoc test between the groups. *, $\mathrm{P}<0.05$ was considered significant.

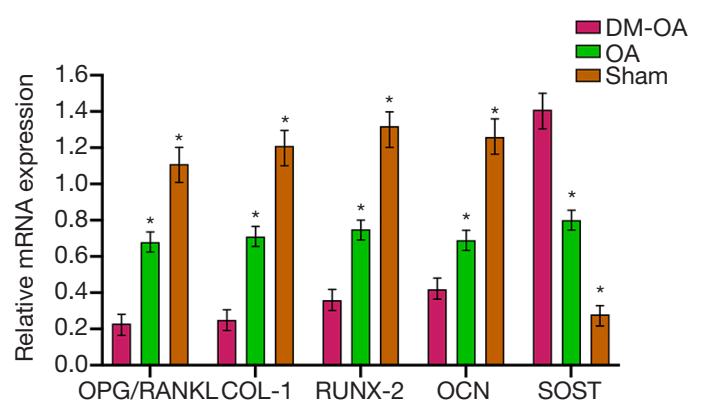

Figure 4 mRNA expression of regulators for bone metabolism in the tibias of mice in the diabetes-osteoarthritis (DM-OA) group, osteoarthritis (OA) group, and sham group at 12 weeks postoperatively. The quantitative results for real-time polymerase chain reaction (RT-PCR). The data are expressed as the mean \pm SD and were compared using ANOVA and the Student-NewmanKeuls post-hoc test between the groups. *, $\mathrm{P}<0.05$ was considered significant. OPG, osteoprotegerin; RANKL, receptor activator of nuclear factor kappa-B ligand; COL-I, collagen type I; RUNX-2, Runt-related transcription factor 2; OCN, osteocalcin; SOST, sclerostin.

(Figures 6,7), cartilage matrix and chondrocyte loss occurred and worsened in the DM-OA group compared to the OA group in a time-dependent manner from 4 to 12 weeks postoperatively. However, the sham group showed no obvious changes at three time points.

At 4 weeks, the DM-OA group and OA group joints exhibited minor cartilage degeneration; matrix and chondrocyte loss affected the superficial and uppermiddle zones. The histological score was not statistically different. Subsequently, at 8 weeks, moderate articular cartilage degeneration reached the middle or deep zone and extended more substantially into the cartilage. The cartilage histological scores of the DM-OA and OA groups were significantly higher than the sham group. At 12 weeks, severe cartilage degeneration was more widespread; matrix and chondrocyte loss deepened to the tidemark. At this stage, the DM-OA group's cartilage histological score was significantly higher than the OA and sham groups.

\section{Micro-CT imaging and analysis}

The DM-OA group had a significant decrease in SB mass and rapid destruction of SB trabecular architecture Representative three-dimensional pictures and morphometric parameters of the tibial SB from the three groups were obtained by micro-CT scanning. These confirmed that DM deteriorated the trabecular bone architecture and accelerated OA progression (Figures 8,9). Generally, tibial SB showed dynamic alterations following $\mathrm{OA}$ induction from bone resorption at the early stages ( 4 to 8 weeks) to bone accretion 

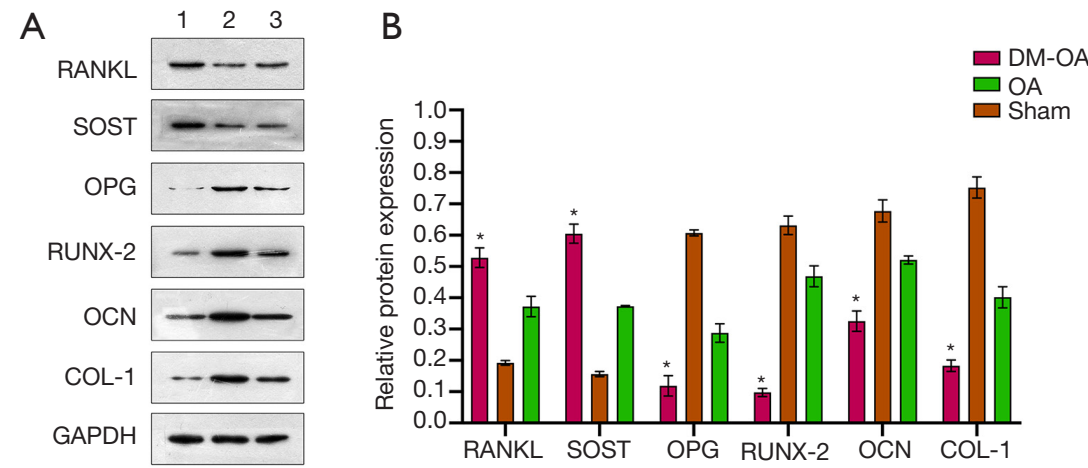

Figure 5 Protein expression of regulators for bone metabolism in mice's tibias in the diabetes-osteoarthritis (DM-OA) group, osteoarthritis (OA) group, and sham group at 12 weeks postoperatively. (A) Immunoblotting bands; (B) the quantitative results for targeted proteins. The data were expressed as the mean \pm SD and were compared using ANOVA and the Student-Newman-Keuls post-hoc test. * $\mathrm{P}<0.05$ was considered significant. OPG, osteoprotegerin; RANKL, receptor activator of nuclear factor kappa-B ligand; COL-I, collagen type I; RUNX-2, Runt-related transcription factor 2; OCN, osteocalcin; SOST, sclerostin.
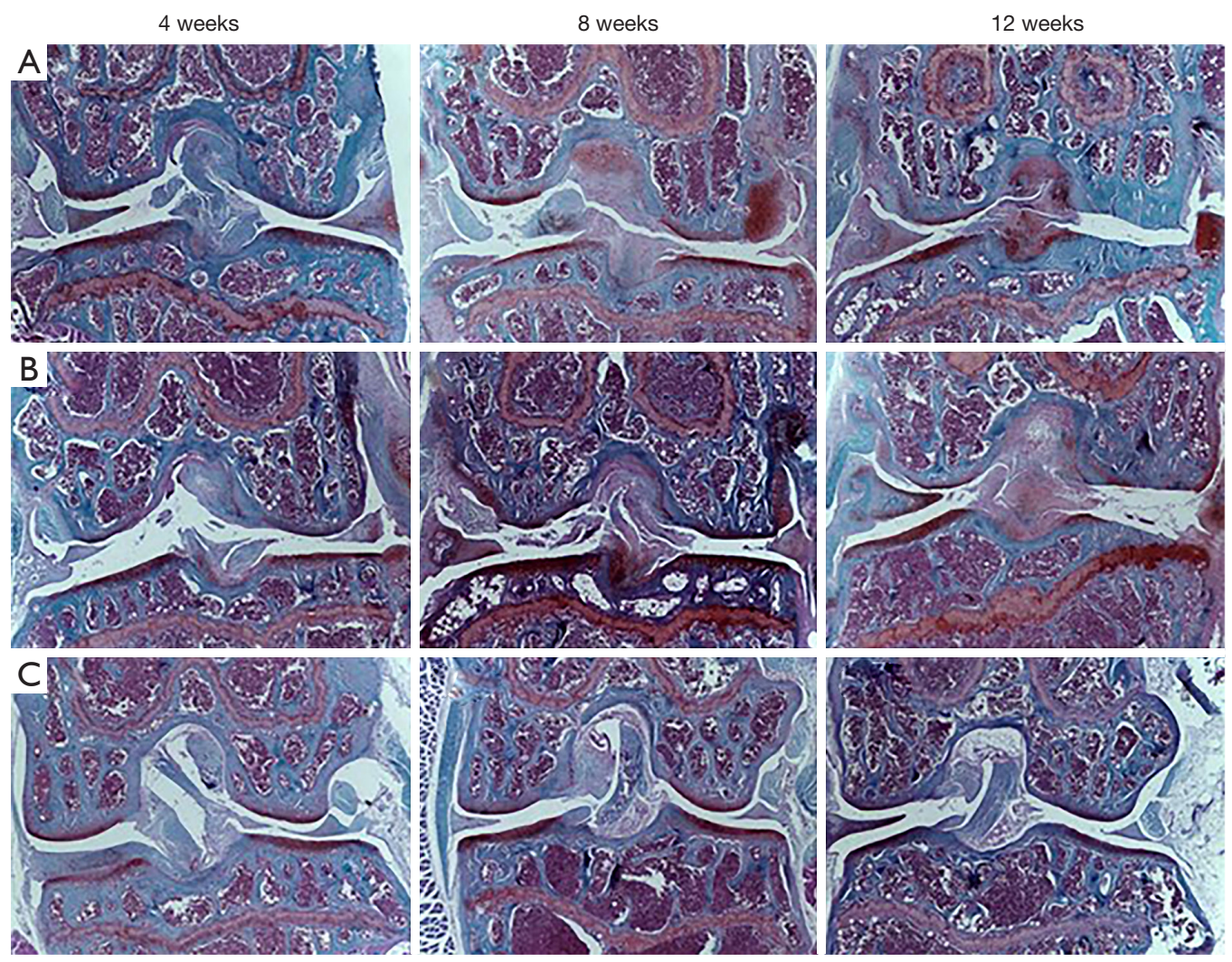

Figure 6 Cartilage degeneration during diabetes mellitus and osteoarthritis (OA) progression (safranin-O/fast green staining). Histology images of the knee joint from the diabetes-osteoarthritis (DM-OA) group (A), OA group (B), and sham group (C) at each time point of sacrifice (4, 8, and 12 weeks postoperatively). Grading of histological sections revealed more serious deterioration of OA's articular cartilage and progression in the DM-OA group compared to the OA and sham groups. Specifically, we observed focal loss of safranin-O staining, indicating decreased proteoglycan concentration, fissuring of articular cartilage, frequent loss and the flattened elongated chondrocytes, cell death in the articular cartilage, and atrophy of articular chondrocytes. 


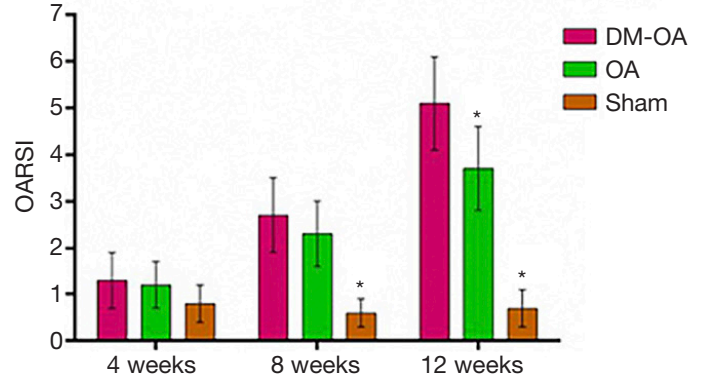

Figure 7 Histological scores of the knee joint from the diabetesosteoarthritis (DM-OA) group, osteoarthritis (OA) group, and sham group at 4,8 , and 12 weeks postoperatively, as determined using the Osteoarthritis Research Society International (OARSI) scoring system. Grading of OA using the OARSI scale showed a statistically significant OA progression in the DM-OA group compared to the OA and sham groups. The data were expressed as the mean $\pm \mathrm{SD}$ and were compared using ANOVA and the Student-Newman-Keuls post-hoc test between the groups. *, $\mathrm{P}<0.05$ was considered significant. at the advanced stages ( 8 to 12 weeks) in the OA group. In contrast, DM-induced significant losses, and trabecular bone resorption resulted in the destruction of SB structure in the DM-OA group throughout the process. Specifically, compared with the sham group, the BV/TV, Tb.Th, and $\mathrm{BMD}$ in the OA group decreased from 4 to 8 weeks $(\mathrm{P}>0.05)$, then increased significantly from 8 to 12 weeks $(\mathrm{P}<0.05)$. An increase in the Tb.Sp and structure model index (SMI) accompanied these changes from 4 to 8 weeks $(\mathrm{P}>0.05)$, then Tb.Sp and SMI decreased from 8 to 12 weeks $(\mathrm{P}<0.05)$. On the other hand, there was a greater rise in BV/TV, Tb.Th and $\mathrm{BMD}$ in the $\mathrm{DM}-\mathrm{OA}$ group $(\mathrm{P}<0.05)$. A decline in Tb.sp and SMI paralleled this during the whole process $(\mathrm{P}<0.05)$.

\section{Confocal Raman microspectroscopy}

Declined trabecular bone mineralization in the DM-OA group

Raman spectral parameters of mineral and matrix composition
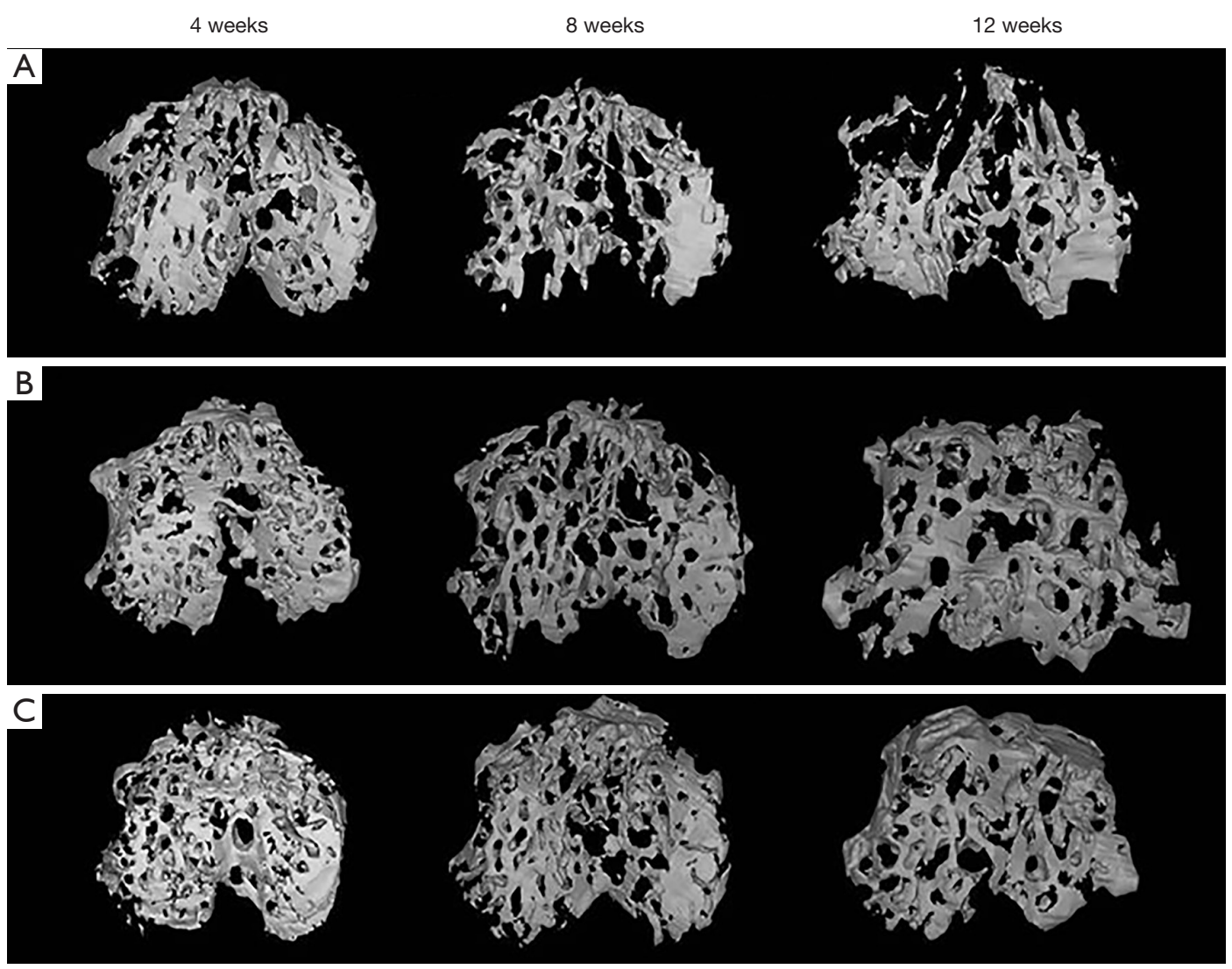

Figure 8 Changes in the subchondral bone microarchitecture of the diabetes-osteoarthritis (DM-OA) group (A), osteoarthritis (OA) group (B), and sham group (C) assessed by micro-CT at each time point of sacrifice $(4,8$, and 12 weeks postoperatively). The DM-OA group exhibited higher trabecular bone mass loss as compared with the OA and sham groups. CT, computed tomography. 

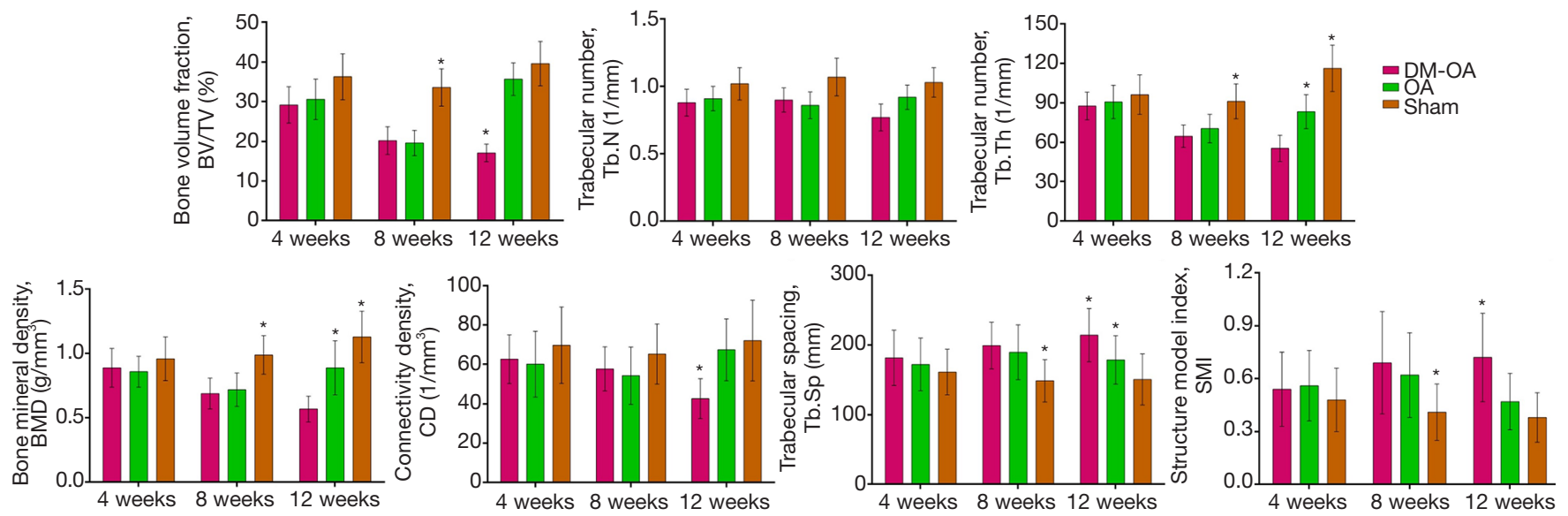

Figure 9 Quantitative analysis includes parameters derived from the geometric microstructure of the tibial subchondral bone (SB). The data were expressed as the mean \pm SD and were compared using ANOVA and the Student-Newman-Keuls post-hoc test between the groups. *, $\mathrm{P}<0.05$ was considered significant. DM-OA, diabetes-osteoarthritis group; OA, osteoarthritis.
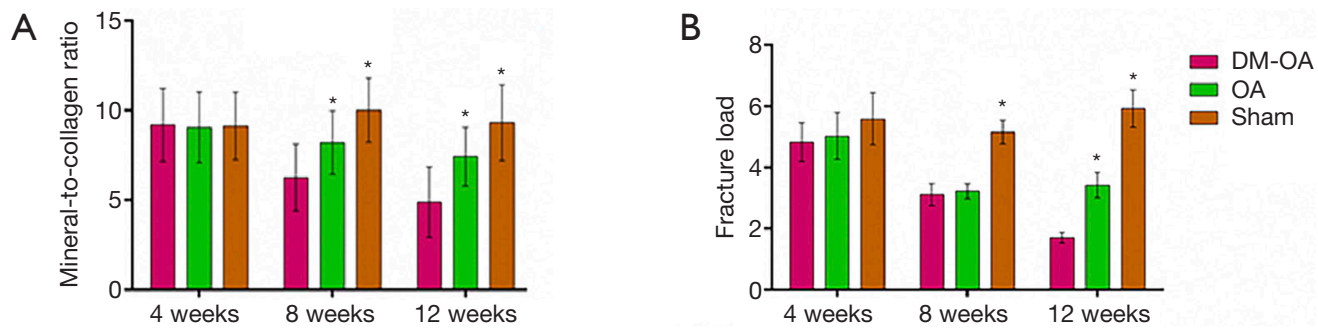

Figure 10 Confocal Raman microspectroscopy and mechanical compression of the subchondral bone (SB). (A) Changes in the mineralto-collagen ratio of the SB among three groups. The mineral-to-collagen ratio was significantly lower in the diabetes-osteoarthritis (DMOA) group than the osteoarthritis (OA) group and sham group at 8 and 12 weeks. (B) Fracture load of SB's compression testing for the three groups at 4, 8, and 12 weeks postoperatively. Graph showing that the fracture load of SB trabecular bone in the DM-OA group was continuously diminished compared to that of the OA and sham groups. The data were expressed as the mean $\pm \mathrm{SD}$ and were compared using ANOVA and the Student-Newman-Keuls post-hoc test between the groups. * $\mathrm{P}<0.05$ was considered significant.

were compared between the three groups to assess DM effects on SB composition (illustrated in Figure 10A). Raman spectra analysis revealed that although no significant difference in mineral-to-collagen ratio was observed at 4 weeks, a reduced mineral-to-collagen ratio was found in the DM-OA and OA groups at 8 and 12 weeks. A significant decline in the DM-OA group was aggravated with the progression of the DM. Specifically, the mineralto-collagen ratio of SB in the DM-OA group was reduced from $9.19 \pm 2.03$ at 4 weeks to $4.89 \pm 1.97$ at 12 weeks. Simultaneously, the mineral-to-collagen ratio of SB in the OA group was reduced from $9.06 \pm 1.96$ at 4 weeks to $7.43 \pm 1.63$ at 12 weeks, whereas in the sham group, the ratio was $9.13 \pm 1.87$ and $9.32 \pm 2.11$, respectively.

\section{Mechanical compression of the SB}

\section{DM-OA group exhibited deterioration of the mechanical properties of SB}

Lastly, the biomechanical properties and strength of SB were assessed using a material testing machine. Although the fracture load difference at 4 weeks was insignificant, there was a significant decrease in the DM-OA and OA groups in 8 weeks. Moreover, the fracture load of SB trabecular bone in the DM-OA group exhibited continuous diminishment compared to that of the $\mathrm{OA}$ and sham groups 

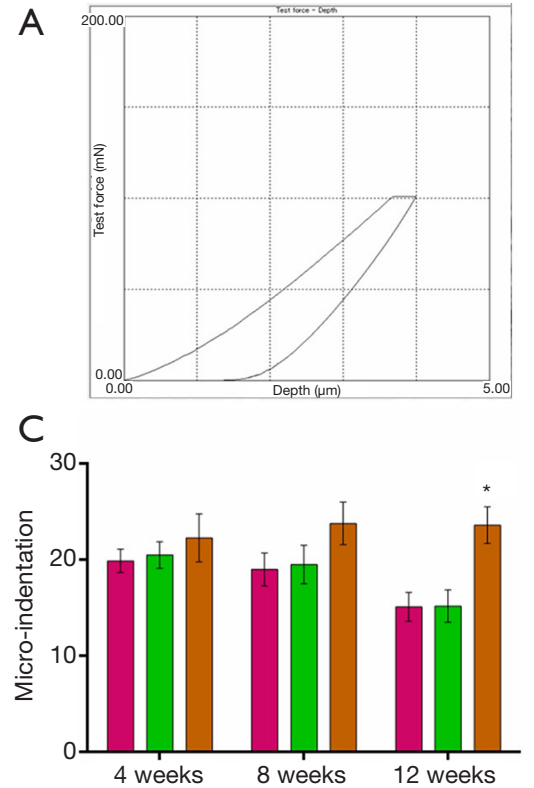
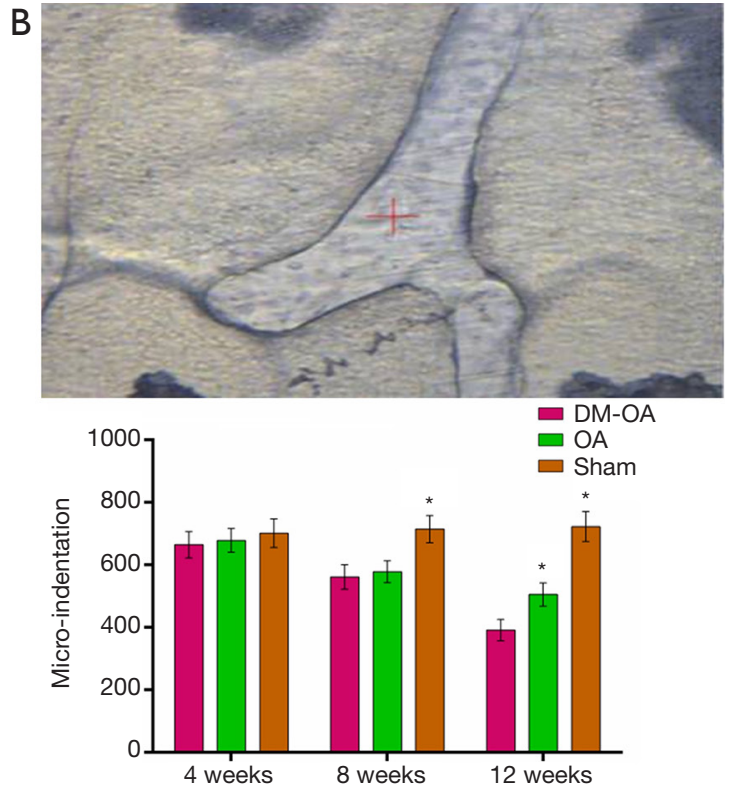

Figure 11 Intrinsic mechanical properties of the subchondral bone trabecular. As early as 8 weeks, alterations in the trabecular bone led to decreased hardness and elastic modulus in the diabetes-osteoarthritis (DM-OA) group. (A) Representative microindentation load-displacement curve from the subchondral bone trabecular; (B) optical microscope image of subchondral bone trabecular showing approximate microindentation positions as a function of measured; (C) results were produced by using the loading protocol for the tissue properties of the mouse bones. The data were expressed as the mean \pm SD and were compared using ANOVA and the Student-NewmanKeuls post-hoc test between the groups. *, $\mathrm{P}<0.05$ was considered significant.

at 12 weeks. As shown in Figure 10B, the fracture load of $\mathrm{SB}$ trabecular bone in the DM-OA group was reduced from $4.83 \pm 0.64 \mathrm{~N}$ at 4 weeks to $1.71 \pm 0.18 \mathrm{~N}$ at 12 weeks.

\section{Microindentation testing}

The DM-OA group had reduced material mechanical strength of the trabecular bone in SB

To further elucidate the intrinsic bone tissue mechanical properties, we investigated the mechanical response of the trabecular bone matrix at the tissue-level displayed as elastic modulus and hardness quantified by microindentation (Figure 11). The SB's mechanical properties in the DM-OA group were statistically reduced compared with that of the OA group as the DM progressed postoperatively. In contrast, there seemed to be no statistically significant differences in the sham group throughout the experiment. Specifically, the elastic modulus of trabecular bone in the DM-OA group was reduced from $19.9 \pm 1.2$ at 4 weeks to $15.1 \pm 1.5$ at 12 weeks. Simultaneously, the mineral-to-collagen ratio of SB in the OA group was reduced from $665.06 \pm 42.27$ at 4 weeks to
$392.25 \pm 34.61$ at 12 weeks.

\section{Discussion}

In this longitudinal study, we accurately quantified and compared the changes to joint derangement at three different time points in three mice groups during the initiation, progression, and end stages (or joint failure) of OA and DM development. More importantly, our study first investigated the SB quantitative change on the architecture remodel, biochemical composition, biomechanics, and physiological function in a DM mouse model. This work successfully demonstrated that DM aggravated OA pathogenesis in a novel DM-OA mice model, which could help clinical and basic scientific research. Furthermore, the results showed the mechanisms through which DM accelerates $\mathrm{OA}$; that is, by damaging and deteriorating the function of $\mathrm{SB}$, including microarchitecture, chemical composition, and biomechanical properties. To our knowledge, this work is the first to describe the pathogenesis of diabetic OA through DM and SB. 
$\mathrm{DM}$ and $\mathrm{OA}$ are the most common coexistent diseases associated with a significant economic and health burden on both an individual and global scale. As early as the 1980s, many doctors and researchers had noticed this phenomenon (28). A burgeoning body of evidence showed that DM adversely affects articular tissues and exacerbates $\mathrm{OA}(8,9)$. Most notably, a 20-year longitudinal cohort study of 927 individuals revealed that DM has an independent role in the pathogenesis and advancement of OA, controlling age, sex, and body mass index (9). Additionally, DM increases the complications and revision rate of arthroplasty procedures (13). Recently, the epidemiological association between $\mathrm{OA}$ and $\mathrm{DM}$ has been further confirmed by two independent meta-analyses, which showed that stronger evidence is accumulating in favor of an independent contribution of DM to the development and progression of OA $(14,15)$. Nevertheless, the effect has not yet been validated in vivo cartilage, but by using clinic followup information and statistical results (16). This study found a higher histological OARSI score in the DM-OA group than that in the OA and sham groups, which is consistent with previous clinical reports.

However, the mechanisms underpinning the connection between both diseases remain unclear (16). By far, most basic science studies focus on the potential mechanisms linked to DM-influenced end-organ joint damage. Mediators such as hyperglycemia, AGEs (advanced glycation end products), sorbitol, adipokines, and cytokines are activated through oxidative, osmotic, and inflammatory mechanisms to produce tissue damage (16). For example, one group found that human OA chondrocytes from donors cultured in high glucose cannot decrease glucose transporter 1 (GLUT1) protein or reduce glucose transport activity than normal chondrocytes. They also found that high glucose conditions favored oxidants' production and promoted matrix catabolism, which accelerated OA progression (29).

Nevertheless, the majority of studies are in vitro experiments with the chondrocytes in a hyperglycemic environment, which are far removed from in vivo studies with the complicated and inherent characteristics (13). Relatively few studies using STZ-induced DM in mice or rats suggested the presence of cartilage damage due to hyperglycemia. Unfortunately, these studies were consistently cross-sectional and showed that slight and mild joint symptoms identified OA's early stages $(8,9)$. In our longitudinal study of approximately 4 months, we accurately quantified and compared the changes to joint derangement at three different time points in three mice groups during the initiation, progression, and end stages (or joint failure) of $\mathrm{OA}$ and $\mathrm{DM}$ development. The present findings first demonstrated that DM aggravated cartilage degeneration and OA pathogenesis occurred in a time-dependent manner in an instability-induced model, which further strengthens the concept of a metabolic component in OA's pathogenesis. Therefore, this validation study sets a precedent for future investigations into possible mechanisms or pharmacological interventions using our longitudinal model.

SB exerts important stress-bearing and structural support function in normal joints merely as a shock absorber and structural girder. SB can buffer approximately $30 \%$ of the intra-articular stress, maintain the joint's matching, and prevent intra-articular stress concentration (3). On the other hand, SB supplies nutrients to cartilage with approximately $50 \%$ of its glucose and oxygen requirements and facilitates removing metabolic waste products via the healthy crosstalk between SB and cartilage, which is also important to maintain the normal functioning of the joint (6). SB manifestations exhibit dynamic remodeling in parallel with (or even preceding) cartilage degradation, including architecture, biochemical composition, biomechanics, and physiological function, which is widely considered a key feature of OA disease progression (7). Therefore, SB is suggested as a key to developing targeted and effective therapeutic strategies to treat $\mathrm{OA}$.

By now, it has been well established that DM extremely and negatively affects bone health, including cortical bone and cancellous bone (5). Eventually, DM could deteriorate bone quality and strength, leading to a drastic rise in fracture risk. In particular, in subjects with T1DM, there is approximately a 6-fold increase in hip fracture risk and a 2-fold increase in vertebral fracture risk compared to individuals without DM (21). Our study concluded that $\mathrm{DM}$ increasing in bone resorption was confirmed by the increased serum level of ALP-B and TRAP-5b and the decreased serum levels of $\mathrm{Ca}$ and $\mathrm{OCN}$. These in vivo pathological changes in the skeletal system demonstrated that DM negatively affects bone health.

Nevertheless, there are minimal reports regarding the impact of DM on SB. Therefore, our study firstly showed quantitative longitudinal data change on the architecture remodel, biochemical composition and biomechanics, and physiological function in a DM mouse model to better reveal the role of SB in the pathogenesis of diabetic OA. Our results fill the research gap between articular cartilage degeneration and SB abnormality, which are the primary concerns in knee OA. Meanwhile, we also detected the 
mRNA and protein expression of key regulators for bone metabolism. At the final time point of our study, the osteoblast-specific biomarkers (OPG/RANKL ratio, COL-I, RUNX-2, OCN) were suppressed, and DM elevated osteoclast-specific biomarkers (SOST). Protein expression was consistent with mRNA.

It is widely accepted that SB's microarchitecture and bone mass is directly related to the mechanical properties and as determinants of physiological function, which exerts important stress-bearing and structural support function (6). Although few studies focus on the SB, abundant research has demonstrated that DM has adverse effects on cancellous bone, leading to microarchitectural deterioration (20). More recent studies employing advanced three-dimensional imaging techniques have confirmed that total volumetric BMD at various skeletal sites, including the femoral neck, lumbar spine, distal radius, and distal tibia, was significantly lower in people with T1DM (22-37\%) compared to individuals without DM (21). MRI studies in young women with childhood-onset T1DM have also shown clear deficits in the tibia's trabecular microarchitecture (30). Similarly, an animal study conducted on rats showed a $60 \%$ reduction in proximal tibial trabecular bone of rats affected by T1DM compared with non-diabetic controls, with an additional $30 \%$ reduction concerning the moments of inertia at the ulnar or femoral diaphysis. These findings were consistently confirmed in other clinic reports (31). This study found lower total and trabecular volumetric BMD and substantial microarchitectural abnormalities, including low Tb.Th, decreased Tb.N, great Tb.Sp, and network inhomogeneity compared with that of the other two groups. The significantly higher SMI indicated that the trabeculae in the DM group were markedly more rod-like compared with non-diabetic groups. Rod-like trabeculae possess lower mechanical strength and stiffness compared to plate-like trabeculae. Clinically, bone loss at the subchondral plate in knee OA patients with hypertension and type $2 \mathrm{DM}$ compared to non-diabetic controls has been documented, consistent with our results (32). These microarchitectural deteriorations were associated with more severe cartilage destruction in the DM-OA group, supporting the paradigm of focal interactions between SB and cartilage in knee OA's pathogenesis.

DM leads to SB loss, microarchitectural deterioration, and decreased trabecular bone mineral mass, including mineral and organic components. Eventually, DM invokes intrinsic material mechanical strength (23). From a pathophysiological aspect, bone metabolism in T1DM patients is characterized by low bone turnover, and in particular, by reduced bone formation due to decreased osteoblastogenesis, low levels of OCN, and reduced mineral apposition rate (33). Additionally, a slow and short osteoblastic cycle is accompanied by decreased bone mineralization (20). The major experimental models of T1DM have shown that diabetic bones display specific bone mineralization defects, including decreased hydroxyapatite crystal perfection, decreased Ca-to-phosphate composition of the ash, and decreased ash content in certain bones (such as the tibial metaphysic) (34).

We found a significantly reduced mineral-to-collagen ratio of SB trabecular in the DM-OA group at 8 and 12 weeks using the confocal Raman microspectroscopy test at the microscopic level. Our result further confirmed reduced mineralization in SB trabecular, which is a good complement and consistent with previous studies in other locations. To reveal the SB trabecular change in the pathogenesis of diabetic OA, we also examined SB trabecular mechanical strength using the microindentation test. The elastic modulus and hardness of SB trabecular were significantly reduced in the DM-OA group. These results were influenced by intrinsic bone material properties, including mineralization and collagen integrity.

The microindentation test has been used in vitro to evaluate variations among different anatomical sites, the effect of pathologies on the mechanical properties of bone structural units, the bone matrix quality close to implants, and the effect of tissue condition (hydrated, dried, or embedded) during the test $(35,36)$. However, to the best of our knowledge, this is the first study demonstrating that DM contributes to reducing SB strength at the macrolevel. Recently, another kind of microindentation using the OsteoProbe technique has gained acceptance as an in vivo research tool for non-invasively estimating bone material strength in humans $(27,37)$. Following local anesthesia, this device creates microindents over the tibial shaft, which provides a bone material strength measure [bone material strength index (BMSi)]. Using this device, Farr et al. found that compared to controls, postmenopausal women with DM had a significantly lower BMSi of $11.7 \%$. This is consistent with our findings on mice and points to impaired bone material properties as a potential cause of the increased fracture risk observed in these patients (38).

In addition to investigating SB trabecular strength, mineral, and organic components at the macro-level, we also studied SB mechanical properties at an apparent level to reflect the combined effects of microarchitecture, 
turnover, damage accumulation (e.g., microfractures), and mineralization. Due to the small size of mice, a 0.8 $\mathrm{mm}$ diameter flat-tipped cylindrical punch was fixed to the actuator of the materials testing machine and compressed directly to record the mechanical properties data. Sankar et al. and numerous other studies developed and improved this method to measure the axial compressive strength of the cancellous bone in the femur of mice, which is more precise and visual than finite element modeling (39-44). The present results in the sham group were consistent with form studies. The differences between previous studies were that we continuously tested at three different time points in our longitudinal study, and the ultimate stress parameter of the DM-OA group was significantly lower than the OA and sham groups. These data further illustrate that DM aggravated cartilage degeneration and $\mathrm{OA}$ pathogenesis by deteriorating SB's stress-bearing and structural support function. The form studies also demonstrated that DM extremely and negatively affects bone quality and strength from various anatomic sites (22). While three-point bending can still be used to study long bones, direct compression is commonly used to measure the spine's cancellous bone mechanical properties (24). Consequently, the fracture risk of DM patients was increased by approximately $70 \%(21)$.

\section{Conclusions}

In conclusion, for the first time, the present study showed that DM aggravated OA pathogenesis in a novel DMOA mouse model, which could help clinical and basic scientific research. More importantly, the results showed the mechanisms through which DM accelerates OA progression; that is, by damaging and deteriorating the functions of SB, including microarchitecture, chemical composition, and biomechanical properties.

\section{Acknowledgments}

The authors would like to thank Dick Ho Kiu Chow and Jia-Li Wang (Musculoskeletal Research Laboratory, Department of Orthopaedics and Traumatology, The Chinese University of Hong Kong, Hong Kong SAR, China), Yu-Long Sun, Xin-Luan Wang, Long-Xiang Lin, Hui-Juan Cao, Ling Li and Ke-Da Shi (Translational Medicine R\&D Center, Institute of Biomedical and Health Engineering, Shenzhen Institutes of Advanced Technology, Chinese Academy of Sciences, Shenzhen, China) who gave technical assistance.
Funding: This work was supported by the Natural Science Foundation of Guangdong Province (grant Number 2016A030313100), the National Natural Science Foundation of China (grant Number 81601219), the China Postdoctoral Science Foundation (grant Numbers 2015M582480 and 2017T100660), the Science and Technology Program of Guangzhou, China (2020324353), and the Medical Scientific Research Foundation of Guangdong Province (A2019100).

\section{Footnote}

Reporting Checklist: The authors have completed the ARRIVE reporting checklist. Available at http://dx.doi. org/10.21037/atm-20-6797

Data Sharing Statement: Available at http://dx.doi. org/10.21037/atm-20-6797

Conflicts of Interest: All authors have completed the ICMJE uniform disclosure form (available at http://dx.doi. org/10.21037/atm-20-6797). The authors have no conflicts of interest to disclose.

Ethical Statement: The authors are accountable for all aspects of the work in ensuring that questions related to the accuracy or integrity of any part of the work are appropriately investigated and resolved. Experiments were performed under a project license (No. 20160223001) granted by the Animal Care and Experiment Committee of Jinan University School of Medicine. All animals were kept in strict accordance with the National Laboratory Animal Management Regulations and guidelines of the Animal Feeding and Ethics Committees of the Experimental Animal Center of Jinan University.

Open Access Statement: This is an Open Access article distributed in accordance with the Creative Commons Attribution-NonCommercial-NoDerivs 4.0 International License (CC BY-NC-ND 4.0), which permits the noncommercial replication and distribution of the article with the strict proviso that no changes or edits are made and the original work is properly cited (including links to both the formal publication through the relevant DOI and the license). See: https://creativecommons.org/licenses/by-nc-nd/4.0/.

\section{References}

1. Mandl LA. Osteoarthritis year in review 2018: clinical. 
Osteoarthritis Cartilage 2019;27:359-64.

2. Hunter DJ, Bierma-Zeinstra S. Osteoarthritis. Lancet 2019;393:1745-59.

3. DeFrate LE, Kim-Wang SY, Englander ZA, et al. Osteoarthritis year in review 2018: mechanics. Osteoarthritis Cartilage 2019;27:392-400.

4. Goldring SR, Goldring MB. Changes in the osteochondral unit during osteoarthritis: structure, function and cartilagebone crosstalk. Nat Rev Rheumatol 2016;12:632-44.

5. Castañeda S, Roman-Blas JA, Largo R, et al. Subchondral bone as a key target for osteoarthritis treatment. Biochem Pharmacol 2012;83:315-23.

6. Findlay DM, Kuliwaba JS. Bone-cartilage crosstalk: a conversation for understanding osteoarthritis. Bone Res 2016;4:16028.

7. Yuan XL, Meng HY, Wang YC, et al. Bone-cartilage interface crosstalk in osteoarthritis: potential pathways and future therapeutic strategies. Osteoarthritis Cartilage 2014;22:1077-89.

8. Nielen JTH, Emans PJ, van den Bemt B, et al. Association of type 2 diabetes mellitus with self-reported knee pain and clinical knee osteoarthritis: The Maastricht Study. Diabetes Metab 2018;44:296-9.

9. Schett G, Kleyer A, Perricone C, et al. Diabetes is an independent predictor for severe osteoarthritis: results from a longitudinal cohort study. Diabetes Care 2013;36:403-9.

10. Puenpatom RA, Victor TW. Increased prevalence of metabolic syndrome in individuals with osteoarthritis: an analysis of NHANES III data. Postgrad Med 2009;121:9-20.

11. Yoshimura N, Muraki S, Oka H, et al. Accumulation of metabolic risk factors such as overweight, hypertension, dyslipidaemia, and impaired glucose tolerance raises the risk of occurrence and progression of knee osteoarthritis: a 3-year follow-up of the ROAD study. Osteoarthritis Cartilage 2012;20:1217-26.

12. Magnusson $\mathrm{K}$, Hagen $\mathrm{KB}$, Østerås $\mathrm{N}$, et al. diabetes is associated with increased hand pain in erosive hand osteoarthritis: data from a population-based study. Arthritis Care Res (Hoboken) 2015;67:187-95.

13. Wada O, Nagai K, Hiyama Y, Nitta S, et al. diabetes is a Risk Factor for Restricted Range of Motion and Poor Clinical Outcome After Total Knee Arthroplasty. J Arthroplasty 2016;31:1933-7.

14. Williams MF, London DA, Husni EM, et al. Type 2 diabetes and osteoarthritis: a systematic review and metaanalysis. J Diabetes Complications 2016;30:944-50.

15. Louati K, Vidal C, Berenbaum F, et al. Association between diabetes mellitus and osteoarthritis: systematic literature review and meta-analysis. RMD Open 2015;1:e000077.

16. Veronese N, Cooper C, Reginster JY, et al. Type 2 diabetes mellitus and osteoarthritis. Semin Arthritis Rheum 2019;49:9-19.

17. Kharroubi AT, Darwish HM. Diabetes mellitus: The epidemic of the century. World J Diabetes 2015;6:850-67.

18. Zheng Y, Ley SH, Hu FB. Global aetiology and epidemiology of type 2 diabetes mellitus and its complications. Nat Rev Endocrinol 2018;14:88-98.

19. $\mathrm{Hu} \mathrm{C}$, Jia W. Diabetes in China: Epidemiology and Genetic Risk Factors and Their Clinical Utility in Personalized Medication. Diabetes 2018;67:3-11.

20. Liu DM, Mosialou I, Liu JM. Bone: Another potential target to treat, prevent and predict diabetes. Diabetes Obes Metab 2018;20:1817-28.

21. Weber DR, Schwartz G. Epidemiology of Skeletal Health in Type 1 Diabetes. Curr Osteoporos Rep 2016;14:327-36.

22. Compston J. Type 2 diabetes mellitus and bone. J Intern Med 2018;283:140-53.

23. Sellmeyer DE, Civitelli R, Hofbauer LC, et al. Skeletal Metabolism, Fracture Risk, and Fracture Outcomes in Type 1 and Type 2 Diabetes. Diabetes 2016;65:1757-66.

24. Napoli N, Chandran M, Pierroz DD, et al. Mechanisms of diabetes mellitus-induced bone fragility. Nat Rev Endocrinol 2017;13:208-19.

25. Chiou CS, Wu CM, Dubey NK, et al. Mechanistic insight into hyaluronic acid and platelet-rich plasmamediated anti-inflammatory and anti-apoptotic activities in osteoarthritic mice. Aging (Albany NY) 2018;10:4152-65.

26. Pritzker KP, Gay S, Jimenez SA, et al. Osteoarthritis cartilage histopathology: grading and staging. Osteoarthritis Cartilage 2006;14:13-29.

27. Herrera S, Diez-Perez A. Clinical experience with microindentation in vivo in humans. Bone 2017;95:175-82.

28. Ladjimi A, Youssef S, Chamakhi S, et al. Rheumatologic manifestations in diabetes. Tunis Med 1985;63:213-19.

29. Laiguillon MC, Courties A, Houard X, et al. Characterization of diabetic osteoarthritic cartilage and role of high glucose environment on chondrocyte activation: toward pathophysiological delineation of diabetes mellitus-related osteoarthritis. Osteoarthritis Cartilage 2015;23:1513-22.

30. Abdalrahaman N, McComb C, Foster JE, et al. Deficits in trabecular bone microarchitecture in young women with type 1 diabetes mellitus. J Bone Miner Res 2015;30:1386-93.

31. Silva MJ, Brodt MD, Lynch MA, et al. Type 1 diabetes in young rats leads to progressive trabecular bone loss, 
cessation of cortical bone growth, and diminished whole bone strength and fatigue life. J Bone Miner Res 2009;24:1618-27.

32. Wen CY, Chen Y, Tang HL, et al. Bone loss at subchondral plate in knee osteoarthritis patients with hypertension and type 2 diabetes mellitus. Osteoarthritis Cartilage 2013;21:1716-23.

33. Palermo A, D'Onofrio L, Buzzetti R, et al. Pathophysiology of Bone Fragility in Patients with Diabetes. Calcif Tissue Int 2017;100:122-32.

34. Al-Hariri M. Sweet Bones: The Pathogenesis of Bone Alteration in Diabetes. J Diabetes Res 2016;2016:6969040.

35. Rubin MR. Skeletal fragility in diabetes. Ann N Y Acad Sci 2017;1402:18-30.

36. Hunt HB, Donnelly E. Bone quality assessment techniques: geometric, compositional, and mechanical characterization from macroscale to nanoscale. Clin Rev Bone Miner Metab 2016;14:133-49.

37. Farr JN, Khosla S. Determinants of bone strength and quality in diabetes mellitus in humans. Bone 2016;82:28-34.

38. Farr JN, Drake MT, Amin S, et al. In vivo assessment of bone quality in postmenopausal women with type 2 diabetes. J Bone Miner Res 2014;29:787-95.

39. Sankar U, Pritchard ZJ, Voor MJ. Micro-computed

Cite this article as: Wang $\mathrm{HJ}$, Giambini $\mathrm{H}$, Chen JW, Wang QS, Hou HG, Luo SM, Chen JY, Zhuang TF, Chen YF, Wu TT, Zha ZG, Liu YJ, Zheng XF. Diabetes mellitus accelerates the progression of osteoarthritis in streptozotocin-induced diabetic mice by deteriorating bone microarchitecture, bone mineral composition, and bone strength of subchondral bone. Ann Transl Med 2021;9(9):768. doi: 10.21037/atm-20-6797 tomography assisted distal femur metaphyseal blunt punch compression for determining trabecular bone strength in mice. J Biomech 2016;49:1233-37.

40. Pritchard ZJ, Cary RL, Yang C, et al. Inhibition of CaMKK2 reverses age-associated decline in bone mass. Bone 2015;75:120-7.

41. Dubrow SA, Hruby PM, Akhter MP. Gender specific LRP5 influences on trabecular bone structure and strength. J Musculoskelet Neuronal Interact 2007;7:16673.

42. Chen Y, Huang YC, Yan CH, et al. Abnormal subchondral bone remodeling and its association with articular cartilage degradation in knees of type 2 diabetes patients. Bone Res 2017;5:17034.

43. van Meurs JB, Boer CG, Lopez-Delgado L, et al. Role of Epigenomics in Bone and Cartilage Disease. J Bone Miner Res 2019;34:215-30.

44. Hunt HB, Torres AM, Palomino PM, et al. Altered Tissue Composition, Microarchitecture, and Mechanical Performance in Cancellous Bone From Men With Type 2 Diabetes Mellitus. J Bone Miner Res 2019;34:1191-206.

(English Language Editor: A. Kassem) 